\title{
ERRATUM: POINT-TO-PERIODIC AND PERIODIC-TO-PERIODIC CONNECTIONS *
}

\author{
LUCA DIECI $^{1}$ and JORGE REBAZA ${ }^{2}$ \\ ${ }^{1}$ School of Mathematics, Georgia Institute of Technology, Atlanta, GA 30332 U.S.A. \\ email: dieci@math.gatech.edu \\ ${ }^{2}$ School of Mathematics, Southwest Missouri State University, Springfield, MO 65804 \\ U.S.A. email: jrebaza@math.smsu.edu
}

Following is a list of some corrections we wanted to make to our work before its publication. Unfortunately, the corrections arrived late to the Publisher and were not accounted for in the paper Point-to-Periodic and Periodic-to-Periodic Connections, which appeared in BIT, 44 (2004), pp. 41-62.

1. P. 44, line 8. Replace [5] with [5, 20].

2. P. 45 , line 18 . Replace $\left[T_{-}, T_{+}\right]$with $J:=\left[T_{-}, T_{+}\right]$.

3. P. 45, lines 23-24. Modify as “... of Beyn's proofs, following [20]. The following lemma ...".

4. P. 45, replace last 4 lines with the following between quotes.

"In the theorems below, we use the spaces

$$
W:=C^{1}\left(J, \mathbb{R}^{m}\right) \times \mathbb{R}^{p} \quad \text { and } \quad Z:=C\left(J, \mathbb{R}^{m}\right) \times \mathbb{R}^{m_{-}^{c}+m_{-}^{s}} \times \mathbb{R}^{m_{+}^{u}+1} .
$$

For appropriate $\alpha, \beta>0$, the norms are defined as

$$
\begin{aligned}
\|(x, \lambda)\|_{W} & =\sup _{t \in J_{-}}\|x(t)\| \mathrm{e}^{\alpha t}+\sup _{t \in J_{+}}\|x(t)\| \mathrm{e}^{-\beta t}+\|\lambda\|, \\
\left\|\left(y, r_{-}, r_{+}\right)\right\|_{Z} & =\left\|\left(y, r_{-}\right)\right\|_{Z_{1}}+\left\|\left(y, r_{+}\right)\right\| z_{2}, \quad \text { where } \\
\left\|\left(y, r_{-}\right)\right\|_{Z_{1}} & =\sup _{t \in J_{-}}\|y(t)\| \mathrm{e}^{\alpha t}+\left\|r_{-}\right\|, \quad \text { and } \\
\left\|\left(y, r_{+}\right)\right\|_{Z_{2}} & =\sup _{t \in J_{+}}\|y(t)\| \mathrm{e}^{-\beta t}+\left\|r_{+}\right\|, \quad\|\cdot\|=\|\cdot\|_{\infty},
\end{aligned}
$$

and where $J_{-}=\left[T_{-}, 0\right]$ and $J_{+}=\left[0, T_{+}\right]$. With these norms, $W$ and $Z$ become Banach spaces. Anticipating the asymptotic convergence of $x(t)$ to $y(t)$ with rate $\epsilon>0$, we impose the condition that, for some constant $C,\left\|x(t)-y_{ \pm}(t)\right\| \leq$ $C \mathrm{e}^{-\epsilon|t|}$ as $t \rightarrow \pm \infty . "$

- As a consequence of the above change, the norms in the statements of Theorems 2.1 and 2.2 need to be changed as well. So, the following changes are needed.

\footnotetext{
* Received June 2004.
} 
5. P. 46, line 6-8. It is now $K_{\delta}=\left\{(x, \lambda):\left\|(x, \lambda)-\left(\left.\bar{x}\right|_{J}, \bar{\lambda}\right)\right\|_{W} \leq \delta\right\}$.

6. P. 46, formula (2.10). The left-hand side changes to

$$
\left\|\left(\left.\bar{x}\right|_{J}, \bar{\lambda}\right)-\left(x_{J}, \lambda_{J}\right)\right\|_{W} .
$$

7. P. 46, line 13. Replace [4, Theorem 3.1] with [20, Theorem 4].

8. P. 46, formula (2.11). The left-hand side changes to

$$
\left\|F\left(\left.\bar{x}\right|_{J}, \bar{\lambda}\right)\right\|_{Z}
$$

9. P. 46, formula (2.12). The left-hand side changes to

$$
\left\|\left(\left.\bar{x}\right|_{J}, \bar{\lambda}\right)-\left(x_{J}, \lambda_{J}\right)\right\|_{W} .
$$

